\title{
On the Number of Guard Edges of a Polygon*
}

\author{
Jung-Heum Park, ${ }^{1}$ Sung Yong Shin, ${ }^{1}$ Kyung-Yong Chwa, ${ }^{1}$ and Tony C. Woo ${ }^{2}$ \\ ${ }^{1}$ Department of Computer Science, Korea Advanced Institute of Science and Technology, \\ Kusong-dong 373-1, Yusong-ku, Taejon 305-701, Republic of Korea \\ \{jhpark, syshin, kychwa\}@gayakum.kaist.ac.kr \\ ${ }^{2}$ Department of Industrial and Operations Engineering, University of Michigan, \\ Ann Arbor, MI 48109, USA \\ Tony.Woo@um.cc.umich.edu
}

\begin{abstract}
This paper establishes tight bounds on the number of edges a polygon from which every point in the polygon is visible; we call them guard edges. For a nonstarshaped polygon, there can be at most three guard edges. For a polygon with holes, there may be at most six; three on the outer boundary and three on one of the holes. The results give new insights into the structure of visibility in polygons and shed light on developing an efficient algorithm for finding all guard edges of a polygon with or without holes.
\end{abstract}

\section{Introduction}

Klee [7] originally posed the art-gallery problem of determining the minimum number of stationary guards so that every point in the interior of an $n$-wall gallery is seen by at least one stationary guard. It is assumed that a guard can see in all directions. The gallery can be modeled as a polygon with $n$ vertices. $\lfloor n / 3\rfloor$ stationary guards were proved to be sufficient and sometimes necessary to guard a polygon [4], [6]. Algorithms for locating the stationary guards were presented $[2],[5]$, and [13].

Variations of this problem were made by considering interior obstacles in the gallery, or by allowing each guard to move along a line segment in the gallery. This guard is called a mobile guard. The gallery with interior obstacles can be

\footnotetext{
* This work was partially supported by the Korea Science and Engineering Foundation (Project No. 91-01-01).
} 


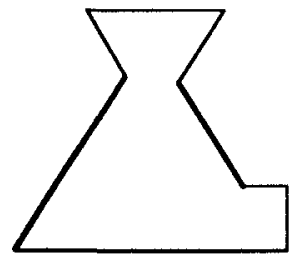

(a)

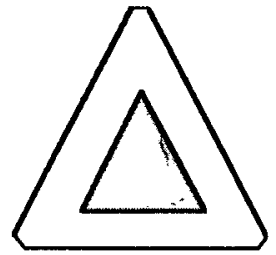

(b)

Fig. 1. (a) A nonstarshaped polygon which has three guard edges. (b) A polygon with holes which has six guard edges; three on the outer boundary and three on one of the holes.

modeled as a polygon with holes. $\lfloor n / 4\rfloor$ mobile diagonal guards are known to be sufficient and sometimes necessary to guard a polygon without holes [10], where mobile diagonal guards are mobile guards capable of moving along a diagonal or an edge. The problem of minimizing the number of stationary guards was proved to be NP-hard [1], [8]. It is also known to be NP-hard to minimize the number of mobile edge guards, mobile guards capable of moving along an edge, in a polygon without holes [8]. An excellent review of these results for art-gallery problems can be found in [11].

An edge is called a guard edge if a mobile edge guard patrolling along the edge can see every point in the polygon. In this paper we show that at most three guard edges exist in a nonstarshaped polygon and that there are at most six guard edges for a polygon with holes; three on the outer boundary and three on one of the holes (see Fig. 1(a) and (b)).

For the problem of finding all guard edges in a polygon, an $O(n)$ algorithm was provided by Shin [15]. This algorithm first determines if the polygon is starshaped in $O(n)$ time using the kernel-finding algorithm in [9]. If the polygon is starshaped, it employs the algorithm in [16]; otherwise, it finds a constant number of candidate guard edges including all guard edges, if any, in $O(n)$ time, and then it determines whether or not a candidate guard edge is indeed a guard edge by employing the algorithm in [3]. Another $O(n)$ algorithm for the problem was presented by Sack and Suri [14].

For the problem of finding all guard edges in a polygon with holes, a similar approach in [15] can be taken. Using the results in Section 4, we can develop an $O(n)$ algorithm for finding a constant number (at most six) of candidate guard edges among which all guard edges can be chosen, if any. To determine whether or not a candidate guard edge is indeed a guard edge, an algorithm for computing the visibility polygon from an edge developed by Suri and O'Rourke [17] can be employed.

\section{Definitions and Notation}

Let $P$ be a simple polygon with $n$ vertices in the plane. We denote the boundary, interior, and exterior of a polygon $P$ by $B d(P), \operatorname{Int}(P)$, and $\operatorname{Ext}(P)$, respectively, so that $P=B d(P) \cup \operatorname{Int}(P)$. Two points $u$ and $v$ in $P$ are said to be visible (with respect 
to $P$ ) if the line segment $L(u, v)$ joining $u$ and $v$ is completely contained in $P$. $P$ is said to be line-visible from $L(u, v)$ in $P$ if, for every point $s$ in $P$, a point $t$ in $L(u, v)$ (depending on $s$ ) exists such that $s$ and $t$ are visible.

Two points $u$ and $v$ in $B d(P) \cup \operatorname{Ext}(P)$ is said to be externally visible if the line segment $L(u, v)$ is completely contained in $B d(P) \cup \operatorname{Ext}(P)$. $P$ is said to be externally line-visible from $L(u, v)$ in $B d(P) \cup \operatorname{Ext}(P)$ if, for every point $s$ in $B d(P)$, a point $t$ in $L(u, v)$ exists such that $s$ and $t$ are externally visible. If such a line segment $L(u, v)$ is an edge $E(u, v)$ of $P$, then $P$ is said to be externally edge-visible from $E(u, v)$.

Let $\left[P ; H_{1}, \ldots, H_{h}\right]$ denote the polygon $P$ with $h$ holes $H_{1}, H_{2}, \ldots, H_{h}$. $\left[P ; H_{1}, \ldots, H_{h}\right]$ is called well formed if $H_{i} \subseteq \operatorname{Int}(P)$ for every hole $H_{i}$, and $H_{i} \cap H_{j}=\varnothing$ for every pair of holes $H_{i}$ and $H_{j}, i \neq j$. A simple polygon $P$ without holes is denoted by $[P ; \varnothing]$. We define the boundary, interior, and exterior of $\left[P ; H_{1}, \ldots, H_{h}\right]$ as follows:

$$
\begin{gathered}
B d\left(\left[P ; H_{1}, \ldots, H_{h}\right]\right)=B d(P) \cup \bigcup_{i=1}^{h} B d\left(H_{i}\right), \\
\operatorname{Int}\left(\left[P ; H_{1}, \ldots, H_{h}\right]\right)=\operatorname{Int}(P)-\bigcup_{i=1}^{h} H_{i},
\end{gathered}
$$

and

$$
\operatorname{Ext}\left(\left[P ; H_{1}, \ldots, H_{h}\right]\right)=\operatorname{Ext}(P) \cup \bigcup_{i=1}^{h} \operatorname{Int}\left(H_{i}\right)
$$

For a polygon $\left[P ; H_{1}, \ldots, H_{h}\right]$ with holes, the notion of visibility can be defined similarly.

\section{Guard Edges of a Nonstarshaped Polygon}

Let $P$ be a simple polygon with $n$ vertices. Starting from a vertex $v_{0}$ and traversing the boundary $B d(P)$ of $P$ in the counterclockwise order, we label the $j$ th vertex from $v_{0}$ as $v_{i}$, where $i$ is taken $j$ modulo $n$. The visibility polygon $V(q, P)$ from $q$ is the set of all points in $P$ which is visible from $q$. The kernel $K(P)$ of $P$ is the set of all points $q$ in $P$ such that $V(q, P)=P$.

In this section we show that at most three guard edges exist in a nonstarshaped polygon, which is defined to be a polygon whose kernel is empty. We employ Helly's theorem [12].

Lemma 1. Let $\left\{S_{i} \mid i \in I\right.$, where $I$ is an index set $\}$ be a finite collection of $d$ dimensional convex sets. If every subcollection consisting of $d+1$ or fewer sets in the collection has a nonempty intersection, then the entire collection has a nonempty intersection. 
It is well known that $K(P)=\bigcap_{i=0}^{n-1} H P\left(v_{i}, v_{i+1}\right)$, where $H P(p, q)$ denotes the half-plane which lies to the left of $L(p, q)$ when we traverse the line segment from $p$ to $q$. Suppose that $K(P)=\varnothing$. Since $H P\left(v_{i}, v_{i+1}\right), 0 \leq i<n$, is a two-dimensional convex set, the following result is immediate from Lemma 1.

Lemma 2. If $K(P)=\varnothing$, then three distinct edges $E\left(v_{a}, v_{a+1}\right), E\left(v_{b}, v_{b+1}\right)$, and $E\left(v_{c}, v_{c+1}\right)$ exist such that $H P\left(v_{a}, v_{a+1}\right) \cap H P\left(v_{b}, v_{b+1}\right) \cap H P\left(v_{c}, v_{c+1}\right)=\varnothing$.

Assuming that $K(P)=\varnothing$, consider three distinct edges $E\left(v_{a}, v_{a+1}\right), E\left(v_{b}, v_{b+1}\right)$, and $E\left(v_{c}, v_{c+1}\right)$ such that $H P\left(v_{a}, v_{a+1}\right) \cap H P\left(v_{b}, v_{b+1}\right) \cap H P\left(v_{c}, v_{c+1}\right)=\varnothing$. Let $e$ be a guard edge. Then any point $q_{i}$ on $E\left(v_{i}, v_{i+1}\right), i=a, b, c$, is visible from some point on $e$. In particular, each midpoint $m_{i}$ of $E\left(v_{i}, v_{i+1}\right), i=a, b, c$, is visible from $e$. It is obvious that a guard edge shares at least a point with each of three visibility polygons $V\left(m_{i}, P\right), i=a, b, c$.

Since $V\left(m_{i}, P\right) \subseteq H P\left(v_{i}, v_{i+1}\right), i=a, b, c, V\left(m_{a}, P\right) \cap V\left(m_{b}, P\right) \cap V\left(m_{c}, P\right)=\varnothing$. There are two possibilities:

Case 1. Among three visibility polygons, $V\left(m_{i}, P\right), i=a, b, c$, a pair of them which do not have a common intersection exist.

Case 2 . The three visibility polygons are pairwise inersecting.

For each of these cases, we show that there are at most three guard edges. Let us consider Case 1 first. We denote by $\tilde{L}(u, v)$ the open line segment excluding its two endpoints $u$ and $v$.

Lemma 3. In Case 1 at most two guard edges exist.

Proof. Let $V\left(m_{a}, P\right) \cap V\left(m_{b}, P\right)=\varnothing$ without loss of generality. Suppose that at least three guard edges $e_{1}, e_{2}, e_{3}, \ldots$ exist. Each guard edge $e_{k}$ necessarily intersects with $V\left(m_{a}, P\right)$ and $V\left(m_{b}, P\right)$. We choose the closest pair of points, $q_{k}^{a}$ and $q_{k}^{b}$, on each guard edge $e_{k}$ such that $q_{k}^{a} \in V\left(m_{a}, P\right)$ and $q_{k}^{b} \in V\left(m_{b}, P\right)$. No pair of open line segments in $\left\{\tilde{L}\left(q_{1}^{a}, q_{1}^{b}\right), \tilde{L}\left(q_{2}^{a}, q_{2}^{b}\right), \tilde{L}\left(q_{3}^{a}, q_{3}^{b}\right)\right\}$ are interesting, since the intersection of two edges is empty or is one of their endpoints. Thus, three guard edges $e_{1}, e_{2}$, and $e_{3}$ with the following properties exist (see Fig. 2):

Let $C_{a}$ be any continuous path from $q_{1}^{a}$ to $q_{2}^{a}$ such that $C_{a} \subseteq V\left(m_{a}, P\right)$. Similarly, let $C_{b}$ be any continuous path from $q_{1}^{b}$ to $q_{2}^{b}$ such that $C_{b} \subseteq V\left(m_{b}, P\right)$. There is a point $z$ on the open line segment $\tilde{L}\left(q_{3}^{a}, q_{3}^{b}\right)$, which is properly surrounded by (surrounded by and is not on) the closed continuous path $C$ which consists of $C_{a}, L\left(a_{2}^{a}, q_{2}^{b}\right), C_{b}$, and $L\left(q_{1}^{b}, q_{1}^{a}\right)$.

Note that every point on $C$ is in $P$. Since $z$ is surrounded by $C$ and is not on $C$, a positive number $\varepsilon$ exists such that an open disk $D(z, \varepsilon)=\{p \mid$ distance between $z$ and $p$ is less than $\varepsilon\}$ is also surrounded by $C$. Since $z$ is a point on an edge of $P$, $D(z, \varepsilon)$ contains a point in $\operatorname{Ext}(P)$, which is a contradiction of the fact that every point surrounded by $C$ is also in $P$. 


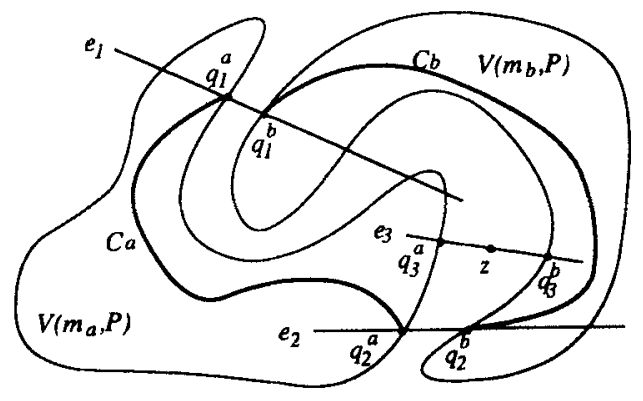

Fig. 2. Illustration of the proof of Lemma 3.

Now consider Case 2, where $V\left(m_{i}, P\right), i=a, b, c$, are pairwise intersecting. By assumption, $H P\left(v_{a}, v_{a+1}\right) \cap H P\left(v_{b}, v_{b+1}\right) \cap H P\left(v_{c}, v_{c+1}\right)=\varnothing$. Since $V\left(m_{i}, P\right) \subseteq$ $H P\left(v_{i}, v_{i+1}\right)$ for all $i=a, b, c, H P\left(v_{i}, v_{i+1}\right), i=a, b, c$, are pairwise intersecting. Let $L_{i}$ be the line containing $E\left(v_{i}, v_{i+1}\right), i=a, b, c$. Let $x_{i j}$ be the intersection point of lines $L_{i}$ and $L_{j}, i \neq j$. It is easy to see the following:

(1) $x_{a b}, x_{b c}$, and $x_{c a}$ are well defined and distinct.

(2) $x_{a b}, x_{b c}$, and $x_{c a}$ are not collinear.

Lemma 4. In Case 2 there are at most three guard edges.

Proof. Suppose for a contradiction that there are four guard edges $e_{1}, e_{2}, e_{3}$, and $e_{4}$ in $P$. We construct a closed continuous path $C$ with the following properties:

(a) Every point on $C$ is in $P$.

(b) There is a point $z$ on one of the guard edges, which is properly surrounded by $C$.

Property (b) implies that a positive number $\varepsilon$ exists such that an open disk $D(z, \varepsilon)=\{p \mid$ distance between $z$ and $p$ is less than $\varepsilon\}$ is properly surrounded by $C$, and that $D(z, \varepsilon)$ contains a point in $\operatorname{Ext}(P)$. This leads to a contradiction of the fact implied by (a) that every point surrounded by $C$ is also in $P$.

First we show that the triangle $T=\left(x_{a b} x_{b c} x_{c a}\right)$ is entirely contained in $P$ by constructing a closed continuous path $C^{\prime}$ such that every point on $C^{\prime}$ is in $P$ and $C^{\prime}$ surrounds $T$. We choose three points $u_{a b}, u_{b c}$, and $u_{c a}$ such that $u_{a b} \in V\left(m_{a}, P\right) \cap$ $V\left(m_{b}, P\right), u_{b c} \in V\left(m_{b}, P\right) \cap V\left(m_{c}, P\right)$, and $u_{c a} \in V\left(m_{c}, P\right) \cap V\left(m_{a}, P\right)$. We construct a continuous path $C_{a}^{\prime}$ from $u_{a b}$ to $u_{c a}$ such that $C_{a}^{\prime} \subseteq V\left(m_{a}, P\right)$ (see Fig. 3(a)). Similarly, we can construct two continuous paths $C_{b}^{\prime}$ and $C_{c}^{\prime}$. Moreover, these three continuous paths can be constructed so that they pairwise intersect only at $u_{a b}$, $u_{b c}$, and $u_{c a}$. Let $C^{\prime}$ denote the closed continuous path which consists of $C_{a}^{\prime}, C_{b}^{\prime}$, and $C_{c}^{\prime}$. By the way in which $C^{\prime}$ is constructed, every point on $C^{\prime}$ is in $P$. $C^{\prime}$ surrounds $T$ since $C_{i}^{\prime} \subseteq H P\left(v_{i}, v_{i+1}\right)$, and $u_{a b} \in I_{a b}, u_{b c} \in I_{b c}, u_{c a} \in I_{c a}$, where $I_{i j}=H P\left(v_{i}, v_{i+1}\right) \cap H P\left(v_{j}, v_{j+1}\right)$ for $i \neq j$. 


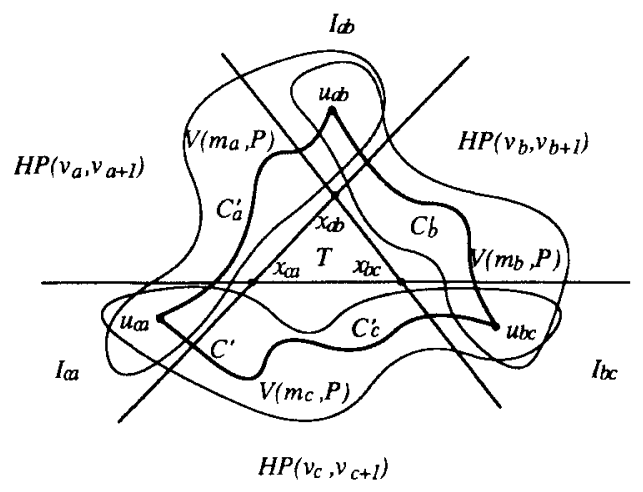

(a)

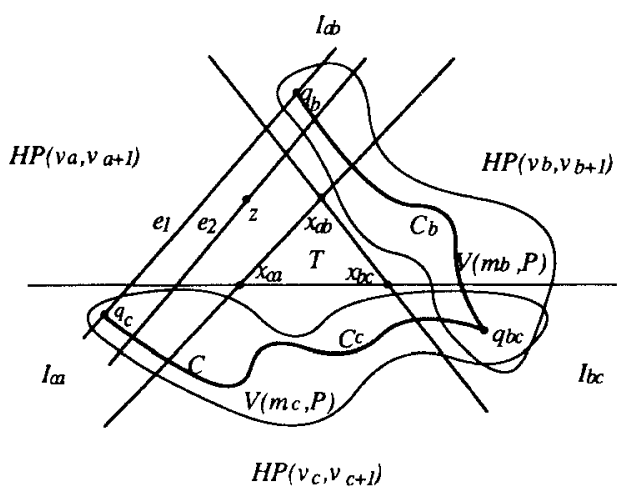

(b)

Fig. 3. Illustration of the proof of Lemma 4.

Each guard edge intersects with exactly two elements of $\left\{I_{a b}, I_{b c}, I_{c a}\right\}$ since

(i) each guard edge $e_{k}$ does not intersect $\operatorname{Int}(T)$,

(ii) each guard edge $e_{k}$ necessarily intersects with $V\left(m_{i}, P\right)$ for all $i=a, b, c$, and thus $e_{k}$ also intersects with $H P\left(v_{i}, v_{i+1}\right)$ for all $i=a, b, c$, and

(iii) there is no guard edge which intersects with all of $\left\{I_{a b}, I_{b c}, I_{c a}\right\}$ (see Fig. $3(\mathrm{~b}))$.

We assume that there are at least four guard edges, thus two guard edges $e_{1}$ and $e_{2}$ with identical type exist, that is, both $e_{1}$ and $e_{2}$ intersect with, say $I_{a b}$ and $I_{c a}$. Without loss of generality, we assume that $e_{1}$ is the last edge encountered when we traverse on the ray starting from a point $x_{b c}$ in the direction of the midpoint of $L\left(x_{a b}, x_{c a}\right)$.

Let $q_{b}$ and $q_{c}$ be the closest pair of points on $e_{1}$ such that $q_{b} \in V\left(m_{b}, P\right)$ and $q_{c} \in V\left(m_{c}, P\right)$, and let $q_{b c}$ be a point in $V\left(m_{b}, P\right) \cap V\left(m_{c}, P\right)$. Since $\left\{q_{b}, q_{b c}\right\} \subseteq$ $V\left(m_{b}, P\right)$, we can construct a continuous path $C_{b}$ from $q_{b}$ to $q_{b c}$ such that 
$C_{b} \subseteq V\left(m_{b}, P\right)$. Similarly, we can also construct a continuous path $C_{c}$ from $q_{c}$ to $q_{b c}$ such that $C_{c} \subseteq V\left(m_{c}, P\right)$. Moreover, the two continuous path $C_{b}$ and $C_{c}$ can be constructed so that $C_{b} \cap C_{c}=\left\{q_{b c}\right\}$. Let $C$ be the closed continuous path which consists of $L\left(q_{b}, q_{c}\right), C_{c}$, and $C_{b}$. By the way in which $C$ is constructed, every point on $C$ is in $P$, and a point $z$ on $e_{2}$ such that

$$
z \in H P\left(v_{a}, v_{a+1}\right)-H P\left(v_{b}, v_{b+1}\right)-H P\left(v_{c}, v_{c+1}\right)
$$

is properly surrounded by $C$. This completes the proof.

The following theorem is immediate from Lemmas 3 and 4, and tautologically implies that every polygon with four or more guard edges is starshaped.

Theorem 5. There are at most three guard edges in a nonstarshaped polygon.

\section{Guard Edges of a Polygon with Holes}

An edge of a hole in $\left[P ; H_{1}, \ldots, H_{h}\right]$ is called an inner edge, and an edge which is not in any hole is called an outer edge. In this section we show that at most six guard edges exist in a polygon with holes; three inner guard edges and three outer guard edges. Let $N_{\mathrm{I}}\left(\left[P ; H_{1}, \ldots, H_{h}\right]\right)$ and $N_{\mathrm{O}}\left(\left[P ; H_{1}, \ldots, H_{h}\right]\right)$ denote the numbers of inner guard edges and outer guard edges of $\left[P ; H_{1}, \ldots, H_{h}\right]$, respectively.

We show that for every polygon $\left[P ; H_{1}, \ldots, H_{h}\right]$ with $h$ holes there is a polygon $[P ; H]$ with only one hole such that $N_{\mathrm{I}}([P ; H]) \geq N_{I}\left(\left[P ; H_{1}, \ldots, H_{h}\right]\right)$ and $N_{\mathrm{O}}([P ; H]) \geq N_{\mathrm{o}}\left(\left[P ; H_{1}, \ldots, H_{h}\right]\right)$, and then prove that for every $[P ; H]$ there is a polygon $\left[P ; H^{\prime}\right]$ with one convex hole such that $N_{\mathrm{l}}\left(\left[P ; H^{\prime}\right]\right) \geq N_{\mathrm{I}}([P ; H])$ and $N_{\mathrm{O}}\left(\left[P ; H^{\prime}\right]\right) \geq N_{\mathrm{o}}([P ; H])$. By concentrating on a polygon $\left[P ; H^{\prime}\right]$ with one convex hole, we finally show that both $N_{\mathrm{l}}\left(\left[P ; H^{\prime}\right]\right)$ and $N_{\mathrm{O}}\left(\left[P ; H^{\prime}\right]\right)$ are less than or equal to three.

The outer edges (resp. inner edges) of $\left[P ; H_{1}, \ldots, H_{h}\right]$ are oriented in the counterclockwise sense (resp. clockwise sense), that is, when they are traversed, the interior of $\left[P ; H_{1}, \ldots, H_{h}\right]$ always lies to the left. Given two points $u$ and $v$ on the outer boundary (resp. inner boundary), the chain $\operatorname{Ch}(u, v)$ is the portion of the boundary from $u$ to $v$ as the boundary is traversed in the counterclockwise sense (resp. clockwise sense). We denote by $\widetilde{C h}(u, v)$ the open chain excluding its two endpoints $u$ and $v$.

Avis and Toussaint [3] found the property that a polygon $P$ is visible from an edge $E(u, v)$ if and only if $B d(P)$ is visible from $E(u, v)$. However, this property does not necessarily hold true for a polygon with holes. Figure 4 shows that, for any positive integer $h \geq 2$, there is a polygon with $h$ holes for which this property does not hold. However, this property holds true for a polygon $[P ; H]$ with only one hole, as stated in Theorem 6 .

Theorem 6. $[P ; H]$ is visible from an edge $E(u, v)$ if and only if $B d([P ; H])$ is visible from $E(u, v)$. 


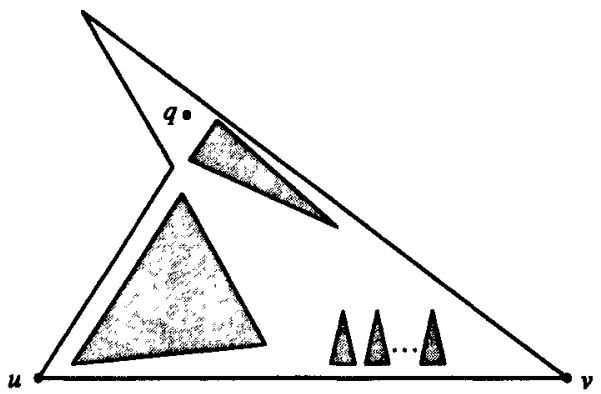

Fig. 4. The boundary of the polygon with $h \geq 2$ holes is visible from the edge $E(u, v)$, but the interior point $q$ is not visible from $E(u, v)$.

Proof. The necessity of the condition is implied by the definitions. For the sufficiency, we show an arbitrary point $q$ in $\operatorname{Int}([P ; H])$ is visible from $E(u, v)$. There are two cases depending on the fact that $E(u, v)$ is an outer or inner edge.

Case 1: $E(u, v)$ is an outer edge. Suppose for a contradiction that $q$ is not visible from $E(u, v)$. The proof of Case 1 consists of two parts. First we choose three points $x, y$, and $z$ on $B d([P ; H])$, and then from the fact that $x, y$, and $z$ are visible from $E(u, v)$, we contradictorily conclude that $q$ is visible from $E(u, v)$. Consider the polygon $[P ; \varnothing]$ which is constructed by removing the hole $H$ from $[P ; H]$. Since $B d([P ; \varnothing])$ is visible from $E(u, v)$ with respect to $[P ; \varnothing],[P ; \varnothing]$ is visible from $E(u, v)$ with respect to $[P ; \varnothing]$. Let $l_{q}$ (resp. $r_{q}$ ) denote the point on $E(u, v)$ farthest from $v$ (resp. from $u$ ) that is visible from $q$ with respect to $[P ; \varnothing]$ (see Fig. 5(a)). Extend $L\left(r_{q}, q\right)$ to the nearest point $x$ on $B d([P ; H])$, and extend $L\left(l_{q}, q\right)$ to the nearest point $y$ on $B d([P ; H])$. Since $q$ is not visible from $E(u, v)$ with respect to $[P ; H]$, there are two points $\alpha \in L\left(q, l_{q}\right)$ and $\beta \in L\left(q, r_{q}\right)$ in $\operatorname{Int}(H)$, and a continuous path $C$ from $\alpha$ to $\beta$ such that $C \subseteq \operatorname{Int}(H)$. Let $z$ be a point on $B d([P ; H])$ such that $z$ lies in the interior of triangle $q \alpha \beta$ and that $z$ is visible from $q$.

Let $x^{\prime}, y^{\prime}$, and $z^{\prime}$ be three points on $E(u, v)$ from which $x, y$, and $z$ are visible, respectively. Extend $L(x, \alpha)$ to a point $x_{\alpha}$ on $E(u, v)$, and extend $L(y, \beta)$ to a point $y_{\beta}$ on $E(u, v)$. By the way in which $l_{q}, r_{q}$, and the continuous path $C$ are constructed, $x^{\prime}$ is on $L\left(l_{q}, x_{\alpha}\right)$ and $y^{\prime}$ is on $L\left(y_{\beta}, r_{q}\right) . z^{\prime}$ is on $L\left(u, l_{q}\right)$ or $L\left(r_{q}, v\right)$, as no points on $L\left(l_{q}, r_{q}\right)$ are visible from $q$. Without loss of generality, we can assume that $z^{\prime}$ is on $L\left(r_{q}, v\right)$. Extend $L(z, \beta)$ to a point $z_{\beta}$ on $E(u, v)$. Then $z^{\prime}$ is on $L\left(z_{\beta}, v\right)$. Now we find a point $q^{\prime} \in E(u, v)$ from which $q$ would be visible. $L\left(y, y^{\prime}\right)$ intersects $L\left(z, z^{\prime}\right)$ at some point $t$ in $[P ; H]$. Consider the quadrilateral $Q=(q z t y)$ whose boundary lies in $[P ; H]$. The hole $H$ is outside $Q$ so that $Q$ lies in $[P ; H]$. Similarly, the triangle $T=\left(t y^{\prime} z^{\prime}\right)$ lies in $[P ; H]$. Extend $L(q, t)$ to a point $q^{\prime}$ on $E(u, v)$. By the way in which $Q$ and $T$ are constructed, $L(q, t)$ lies in $Q$ and $L\left(t, q^{\prime}\right)$ lies in $T$, and thus $q$ is visible from $q^{\prime}$, which is a contradiction.

Case 2: $E(u, v)$ is an inner edge. Extend $E(u, v)$ and $E(v, u)$ to the nearest point $v^{\prime}$ and $u^{\prime}$ on $B d\left([P ; H]\right.$ ), respectively (see Fig. $5(\mathrm{~b})$ ). Both $u^{\prime}$ and $v^{\prime}$ are on the outer 


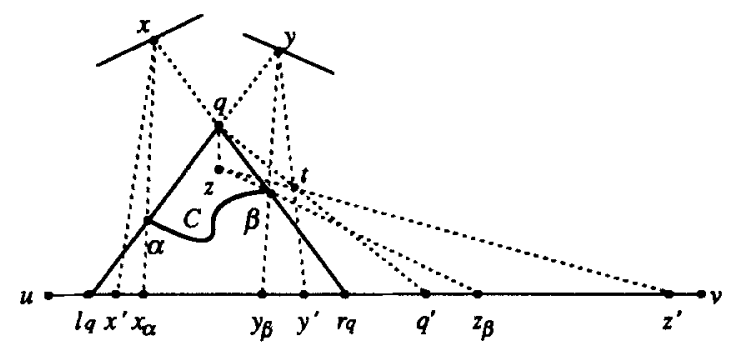

(a)

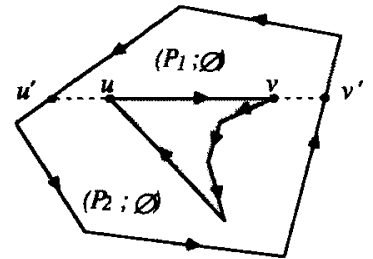

(b)

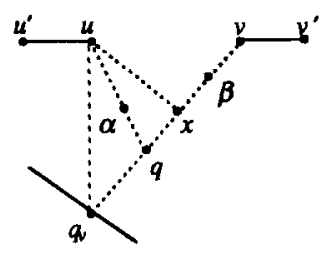

(c)

Fig. 5. Illustration of the proof of Theorem 6: (a) Case 1, (b) and (c) Case 2.

boundary of $[P ; H]$, since the hole $H$ is externally edge-visible from $E(u, v)$. We divide the polygon $[P ; H]$ into two polygons $\left[P_{1} ; \varnothing\right]$ and $\left[P_{2} ; \varnothing\right]$ such that $B d\left(\left[P_{1} ; \varnothing\right]\right)$ consists of $L\left(u^{\prime}, u\right), L(u, v), L\left(v, v^{\prime}\right)$, and $C h\left(v^{\prime}, u^{\prime}\right)$, and $B d\left(\left[P_{2} ; \varnothing\right]\right)$ consists of $C h\left(u^{\prime}, v^{\prime}\right), L\left(v^{\prime}, v\right), C h(v, u)$, and $L\left(u, u^{\prime}\right)$. Now we show that every point in $\left[P_{1} ; \varnothing\right]$ is visible from $E(u, v)$ and every point in $\left[P_{2} ; \varnothing\right]$ is visible from $u$ or $v$.

It is easy to see that every point on $B d\left(\left[P_{1} ; \varnothing\right]\right)$ is visible from $E(u, v)$, and thus every point in $\operatorname{Int}\left(\left[P_{1} ; \varnothing\right]\right)$ is also visible from $E(u, v)$. It remains to show that every point $q$ in $\operatorname{Int}\left(\left[P_{2} ; \varnothing\right]\right)$ is visible from $u$ or $v$. Suppose to the contrary that $q$ is visible from neither $u$ nor $v$. Then two points $\alpha \in L(u, q)$ and $\beta \in L(v, q)$ in $\operatorname{Ext}\left(\left[P_{2} ; \varnothing\right]\right)$ exist (see Fig. 5(c)). Extend $L(v, q)$ to the nearest point $q_{v}$ on $B d\left(\left[P_{2} ; \varnothing\right]\right)$. Let $x \in L(v, q)$ be a point on $B d\left(\left[P_{2} ; \varnothing\right]\right)$ which is visible from $q$. From the fact that neither $q_{v}$ nor $x$ is visible from $v, q_{v}$ and $x$ are visible from $u$. Note that every point in $B d\left(\left[P_{2} ; \varnothing\right]\right)$ is visible from $u$ or $v$. Thus, the boundary of the quadrilateral $Q=\left(u q_{v} q x\right)$ is in $\left[P_{2} ; \varnothing\right]$, but $Q$ contains $\alpha \in \operatorname{Ext}\left(\left[P_{2} ; \varnothing\right]\right)$, which is a contradiction. This completes the proof.

If $e$ is a guard edge of $\left[P ; H_{1}, \ldots, H_{h}\right]$, then all holes are externally line-visible from $e$. A necessary but not sufficient condition for a polygon to be externally line-visible from a line segment $s$ is given in the following lemma.

Lemma 7. Let $s$ be a line segment which does not intersect Int( $P)$. If a polygon $P$ is externally line-visible from $s$, then two supporting lines $l_{1}$ and $l_{2}$ exist which satisfy 


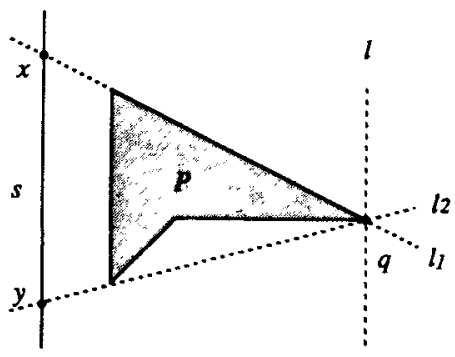

Fig. 6. Illustration of Lemma 7.

the following three conditions (see Fig. 6):

(a) $l_{1}$ and $l_{2}$ pass through an extreme vertex $q$ of $P$ lying on the line $l$ that is parallel to $s$ and lies on the opposite side of $s$ with respect to $P$.

(b) $l_{1}$ and $l_{2}$ intersect $s$ at $x$ and $y$, respectively.

(c) The triangle qxy contains $P$.

Proof. Straightforward.

The following Lemmas 8 and 9 imply that there is a polygon $[P ; H]$ with only one hole such that $N_{\mathrm{I}}([P ; H]) \geq \mathrm{N}_{\mathrm{l}}\left(\left[P ; H_{1}, \ldots, H_{h}\right]\right)$ and $N_{\mathrm{o}}([P ; H]) \geq$ $N_{\mathrm{o}}\left(\left[P ; H_{1}, \ldots, H_{h}\right]\right)$ for any $h \geq 2$. This simplifies our problems for finding the upper bounds on the number of guard edges since we can concentrate on $[P ; H]$ rather than $\left[P ; H_{1}, \ldots, H_{h}\right]$.

Lemma 8. For a polygon with holes, at most one hole has inner guard edges.

Proof. Assume that a hole $H_{i}$ has a guard edge $e$. We show that no other hole $H_{j}$ has a guard edge. Since $H_{j}$ is externally line-visible from $e$, by Lemma 7 , two supporting lines $l_{1}$ and $l_{2}$ pass through an extreme vertex $q$ of $H_{j}$ and intersect $e$ at $x$ and $y$, respectively (see Fig. 7). Draw a line starting at $q$ passing through the midpoint $z$ of $L(x, y)$ to the nearest point $p$ on $B d([P ; H])$. Since $p$ is visible from no point on $B d\left(H_{j}\right), H_{j}$ has no guard edge.

Lemma 9. For a polygon $\left[P ; H_{1}, \ldots, H_{h}\right]$ with $h \geq 2$ holes, $i$ exists, $1 \leq i \leq h$, such that $N_{\mathrm{I}}\left(\left[P ; H_{i}\right]\right) \geq N_{\mathrm{T}}\left(\left[P ; H_{1}, \ldots, H_{h}\right]\right)$ and $N_{\mathrm{o}}\left(\left[P ; H_{i}\right]\right) \geq N_{\mathrm{o}}\left(\left[P ; H_{1}, \ldots, H_{h}\right]\right)$.

Proof. If $\left[P ; H_{1}, \ldots, H_{h}\right]$ has a hole with guard edges, let $H_{i}$ be the hole; otherwise, let $H_{i}$ be any hole of $\left[P ; H_{1}, \ldots, H_{h}\right]$. No guard edge of $\left[P ; H_{1}, \ldots, H_{h}\right]$ is removed when we construct $\left[P ; H_{i}\right]$ by Lemma 8 . The proof is completed if we show that every guard edge of $\left[P ; H_{1}, \ldots, H_{h}\right]$ with respect to $\left[P ; H_{1}, \ldots, H_{h}\right]$ is also a guard edge of $\left[P ; H_{i}\right]$ with respect to $\left[P ; H_{i}\right]$. Let $e$ be a guard edge of $\left[P ; H_{1}, \ldots, H_{h}\right]$ with respect to $\left[P ; H_{1}, \ldots, H_{h}\right]$. Then $B d\left(\left[P ; H_{i}\right]\right) \subseteq$ 


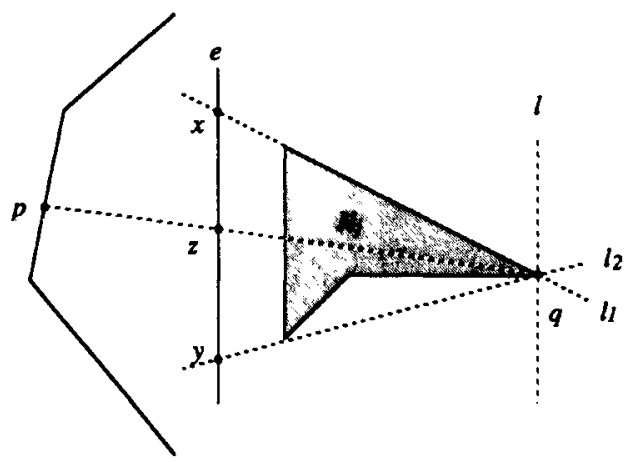

Fig. 7. Illustration of the proof of Lemma 8.

$\mathrm{Bd}\left(\left[P ; H_{1}, \ldots, H_{h}\right]\right)$ is visible from $e$ with respect to $\left[P ; H_{1}, \ldots, H_{h}\right]$. From the fact that $\left[P ; H_{i}\right] \supseteq\left[P ; H_{1}, \ldots, H_{h}\right], B d\left(\left[P ; H_{i}\right]\right)$ is also visible from $e$ with respect to $\left[P ; H_{i}\right]$. Thus, by Theorem $6, e$ is a guard edge of $\left[P ; H_{i}\right]$.

By Lemma 9, we can restrict our attention to the class of polygons with one hole. If an edge $e$ of a hole is an inner guard edge, then the hole is externally edge-visible from $e$. We can further restrict the polygon class by the following two lemmas. The first lemma gives a necessary condition for a polygon without holes to be externally edge-visible from an edge of the polygon.

Lemma 10. If a polygon without holes is externally edge-visible from an edge $e$, then $e$ is an edge of the convex hull of the polygon [15].

Lemma 11. Let $H^{\prime}$ be the convex hull of the hole $H$ of $[P ; H]$. If $\left[P ; H^{\prime}\right]$ is well formed, then $N_{\mathrm{I}}\left(\left[P ; H^{\prime}\right]\right) \geq N_{\mathrm{I}}([P ; H])$ and $N_{\mathrm{o}}\left(\left[P ; H^{\prime}\right]\right) \geq N_{\mathrm{o}}([P ; H])$; otherwise, $[P ; H]$ has no guard edge.

Proof. We assume that $H$ is not convex, for otherwise the result is immediate. Remember that $[P ; H]$ is called well formed if $H \subseteq \operatorname{Int}(P)$.

First, we prove that if $\left[P ; H^{\prime}\right]$ is well formed, then every guard edge of $[P ; H]$ is also a guard edge of $\left[P ; H^{\prime}\right]$, which implies that $N_{\mathrm{I}}\left(\left[P ; H^{\prime}\right]\right) \geq N_{\mathrm{I}}([P ; H])$ and $N_{\mathrm{o}}\left(\left[P ; H^{\prime}\right]\right) \geq N_{\mathrm{o}}([P ; H])$. Let $e$ be an arbitrary guard edge of $[P ; H]$. The guard edge $e$ is an edge of $\left[P ; H^{\prime}\right]$, since every outer edge of $[P ; H]$ is an edge of $\left[P ; H^{\prime}\right]$ and every inner guard edge is an edge of the convex hull $H^{\prime}$ of $H$ by Lemma 10 .

We still need to show that $e$ is a guard edge of $\left[P ; H^{\prime}\right]$ with respect to $\left[P ; H^{\prime}\right]$. Every point $x \in B d\left(\left[P ; H^{\prime}\right]\right)$ is visible from some point $y$ on $e$ with respect to $[P ; H]$, that is, $L(x, y)$ is contained in $[P ; H]$. We show that $L(x, y)$ is contained in $\left[P ; H^{\prime}\right]$. Suppose for a contradiction that a point $\alpha$ on $L(x, y)$ exists such that $\alpha \notin\left[P ; H^{\prime}\right]$. $\alpha$ must be a point in $[P ; H]-\left[P ; H^{\prime}\right]=(P-\operatorname{Int}(H))-\left(P-\operatorname{Int}\left(H^{\prime}\right)\right)=\operatorname{Int}\left(H^{\prime}\right)-$ $\operatorname{Int}(H)$. Let $E(u, v)$ be an edge of $H^{\prime}$ such that the polygon $Q$ consisting of $C h(u, v)$ and $L(v, u)$ contains $\alpha$ (see Fig. 8(a)). Note that $x, y \notin Q-E(u, v)$ and $\alpha \in Q-E(u, v)$. 


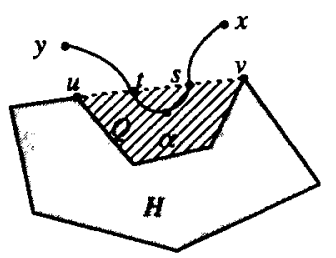

(a)

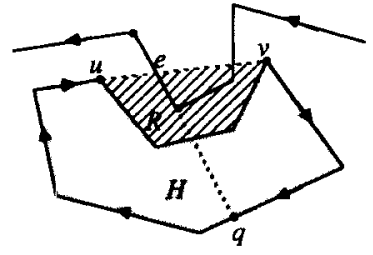

(b)

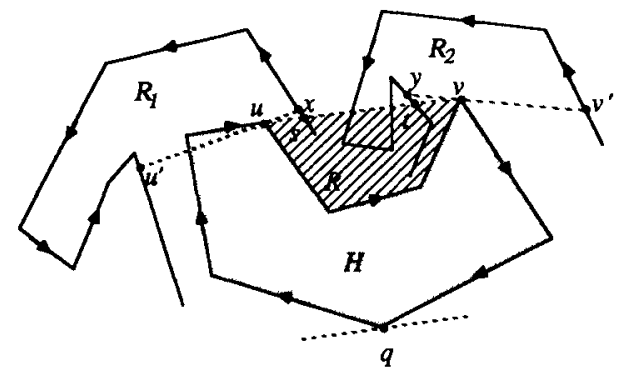

(c)

Fig. 8. Illustration of the proof of Lemma 11.

Therefore, traversing the line segment $L(x, y)$ from $x$ to $y$, we pass through the following three points in sequence: a point $s$ on $E(u, v), \alpha$, and a point $t \neq s$ on $E(u, v)$. This contradicts that $L(x, y)$ is a line segment. Thus, every point on $B d\left[P ; H^{\prime}\right]$ is visible from $e$ with respect to $\left[P ; H^{\prime}\right]$, and $e$ is also a guard edge of $\left[P ; H^{\prime}\right]$ by Theorem 6 .

Second, we prove that if $\left[P ; H^{\prime}\right]$ is not well formed, $[P ; H]$ has no guard edges. By assumption, there is an edge $E(u, v)$ of $H^{\prime}$ which intersects an outer edge of $[P ; H]$. Let $R$ be the polygon whose boundary consists of $C h(u, v)$ and $L(v, u)$ (the hatched polygon in Fig. 8(b) and (c)). Take any edge $e$ in $[P ; H]$. There are two cases depending on the edge $e$.

Case 1: $e$ is an edge of $[P ; H]$ such that $e \cap(R-L(u, v)) \neq \varnothing$. That is, $e$ is an inner edge in $C h(u, v)$ or an outer edge which intersects $R-E(u, v)$. We can see that the line passing through $e$ intersects open chain $\widetilde{C h}(v, u)$ at $q$ (see Fig. 8(b)). $q$ is not visible from $e$, thus $e$ is not a guard edge of $[P ; H]$.

Case 2: $e$ is an edge of $[P ; H]$ such that $e \cap(R-L(u, v))=\varnothing$. That is, $e$ is an inner edge in $C h(v, u)$ or an outer edge which does not intersect $R-E(u, v)$. Suppose for a contradiction that $e$ is a guard edge. We show that $e$ is contained in the line segment $L(u, v)$, which leads to a contradiction because an extreme vertex $q$ of $H$ on the line, which is parallel to $e$ and lies on the opposite side of $e$ with respect to $H$, is not visible from $e$ (see Fig. 8(c)).

Let $s$ (resp. $t$ ) be the first point on $B d([P ; \varnothing])$ which is encountered when we traverse $L(u, v)$ from $u$ to $v$ (resp. from $v$ to $u$ ). Let $x$ (resp. $y$ ) be an arbitrary point 
on $B d([P ; \varnothing])$ such that the length of $C h(s, x)$ (resp. $C h(y, t))$ is less than $\varepsilon$, where $\varepsilon$ is a sufficiently small positive number. Clearly, $x$ (resp. $y$ ) lies on the edge containing $s$ (resp. $t$ ). Let $x^{\prime}$ and $y^{\prime}$ be points on $e$ from which $x$ and $y$ are visible, respectively.

We construct two disjoint polygons $R_{1}$ and $R_{2}$ containing $x^{\prime}$ and $y^{\prime}$, respectively. Extend $L(x, u)$ to the nearest point $u^{\prime}$ on $B d([P ; \varnothing])$, and extend $L(y, v)$ to the nearest point $v^{\prime}$ on $B d([P ; \varnothing])$. We define $R_{1}$ to be the polygon whose boundary consists of $C h\left(s, u^{\prime}\right), L\left(u^{\prime}, u\right)$, and $L(u, s)$, and $R_{2}$ to be the polygon whose boundary consists of $C h\left(v^{\prime}, t\right), L(t, v)$, and $L\left(v, v^{\prime}\right)$. We can always choose $\varepsilon$ so that $R_{1} \cap R_{2}=\varnothing$ and $x^{\prime}$ and $y^{\prime}$ are in $R_{1}$ and $R_{2}$, respectively. Remember that $x^{\prime}$ and $y^{\prime}$ are on $e$, and thus none of them is in $(R-L(u, v))$.

By construction, the line segment $L\left(x^{\prime}, y^{\prime}\right)$ on $e$ lies on the line $l$ containing $L(u, v)$; otherwise, it would intersect $\operatorname{Ext}([P ; H])$. Therefore, the ede $e$ also lies on $l$, and it is contained in $L(u, v)$ since at most one of $u$ and $v$ can be on $e$ as a vertex. This completes the proof.

Now we concentrate on a polygon $[P ; H]$ with one convex hole. From an inner guard edge $e$, the hole $H$ is necessarily externally edge-visible from $e$. The number of externally edge-visible edges of a convex polygon with four or more vertices is zero, because, for any edge $e$ of the polygon, no point on an open edge which is not adjacent to $e$ is visible from $e$. A triangle is externally edge-visible from each of its three edges. Thus, we can conclude that a convex polygon has at most three externally edge-visible edges. This implies that the number of inner guard edges of a polygon $[P ; H]$ with a convex hole is at most three.

Lemma 12. For a polygon $[P ; H]$ with a convex hole, $N_{\mathrm{I}}([P ; H])$ is less than or equal to three, and only a triangular hole has inner guard edges.

Theorem 13. There are at most three inner guard edges in a polygon with holes, and all of them are on one of the holes.

Proof. The result is immediate from Lemmas 8, 9, 11, and 12 .

Now we consider the upper bound on the number of outer guard edges of a polygon with a convex hole.

Lemma 14. For any polygon $[P ; H]$ with a convex hole, $N_{\mathrm{o}}([P ; H])$ is less than or equal to three.

Proof. Suppose for a contradiction that four or more outer guard edges $E\left(u_{i}, v_{i}\right)$ exist, $i=1,2,3,4, \ldots$. Since $H$ is externally line-visible from an outer guard edge $E\left(u_{i}, v_{i}\right), i=1, \ldots, 4$, by Lemma 7 , two supporting lines $l_{i_{1}}$ and $l_{i_{2}}$ pass through an extreme vertex $q_{i}$ of $H$ and intersect $E\left(u_{i}, v_{i}\right)$ at $u_{i}^{\prime}$ and $v_{i}^{\prime}$, respectively (see Fig. 9). Since $H$ is convex, two vertices $q_{i_{1}}$ and $q_{i_{2}}$ of $H$ adjacent to $q_{i}$ lie on the supporting 


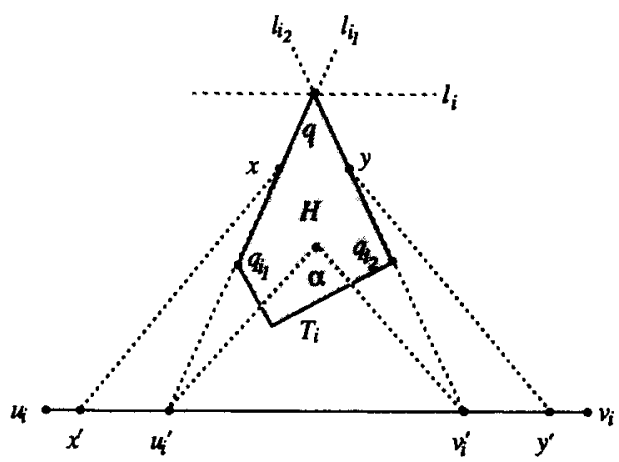

Fig. 9. Illustration of the proof of Lemma 14.

lines $l_{i_{1}}$ and $l_{i_{2}}$, respectively. Let $\alpha$ be a point in $\operatorname{Int}(H)$, and let $T_{i}$ be the triangle $u_{i}^{\prime} \alpha v_{i}^{\prime}$.

We show that (i) $\operatorname{Int}\left(T_{i}\right) \cap \operatorname{Int}\left(T_{j}\right)=\varnothing, i \neq j$, and (ii) $q_{i}$ 's are distinct. Let $x$ and $y$ be the midpoints of $E\left(q_{i}, q_{i_{1}}\right)$ and $E\left(q_{i}, q_{i_{2}}\right)$, respectively. By assumption, $x$ is visible from some point $x^{\prime} \in E\left(u_{i}, v_{i}\right)$, and $y$ is visible from $y^{\prime} \in E\left(u_{i}, v_{i}\right)$. Then $x^{\prime}$ is on $L\left(u_{i}, u_{i}^{\prime}\right)$ and $y^{\prime}$ is on $L\left(v_{i}^{\prime}, v_{i}\right)$. The triangle $T_{i}$ lies in the pentagon $Q_{i}=\left(q_{i} x x^{\prime} y^{\prime} y\right)$ whose boundary lies in $[P ; H]$. Therefore, the open line segments $\tilde{L}\left(u_{i}^{\prime}, \alpha\right)$ and $\tilde{L}\left(v_{i}^{\prime}, \alpha\right)$ intersect no outer edge, which directly implies (i) and (ii).

The sum of internal angles at four distinct extreme vertices of $H$ is

$$
\sum_{i=1}^{4} \angle q_{i_{1}} q_{i} q_{i_{2}}=\sum_{i=1}^{4} \angle u_{i}^{\prime} q_{i} v_{i}^{\prime}<\sum_{i=1}^{4} \angle u_{i}^{\prime} \alpha v_{i}^{\prime} \leq 360^{\circ}
$$

This is a contradiction of the fact that the sum of internal angles of any four distinct vertices of a convex polygon is at least $360^{\circ}$.

Theorem 15. There are at most three outer guard edges in a polygon with holes.

Proof. The proof is immediate from the Lemmas 9, 11, and 14.

Remark. If we consider only polygons with two or more holes, we can get a stronger result: the upper bound on the number of outer guard edges remains three (see Fig. 10(a)), but the number of inner guard edges are at most two (see Fig. 10(b) and Theorem 16). The proof of Theorem 16 is omitted.

Theorem 16. There are at most two inner guard edges in a polygon with two or more holes. 


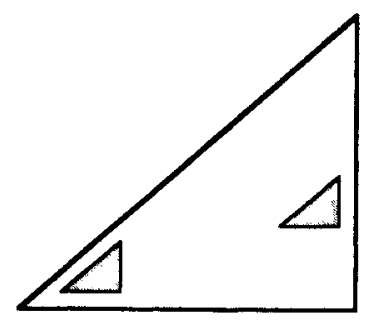

(a)

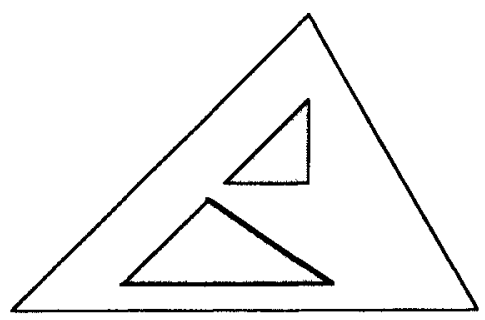

(b)

Fig. 10. A polygon with two holes which has (a) three outer guard edges and (b) two inner guard edges.

\section{Conclusions}

We show the following tight upper bounds on the number of guard edges a polygon: there are at most three guard edges in a nonstarshaped polygon, and there are at most six guard edges in a polygon with holes: three on the outer boundary, and three on one of the holes. The inner guard edge, if any, is an edge of a hole whose convex hull is a triangle. For a polygon with two or more holes, there are at most two inner guard edges. These results may give new insights into the structure of visibility in polygons and a clue for designing an efficient algorithm for finding all guard edges of a polygon with or without holes.

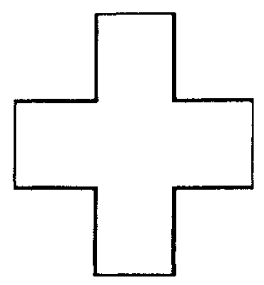

(a)

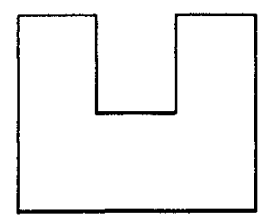

(b)

Fig. 11. (a) A rectilinear polygon which has eight guard edges. (b) A rectilinear nonstarshaped polygon which has two guard edges. 
If we restrict our attention to a rectilinear polygon without holes, we can obtain another upper bound. It is not difficult to see that a rectilinear starshaped polygon has at most eight guard edges (see Fig. 11(a)). If the kernel of a rectilinear polygon $P$ is empty, then there are two edges $E\left(v_{a}, v_{a+1}\right)$ and $E\left(v_{b}, v_{b+1}\right)$ such that $H P\left(v_{a}, v_{a+1}\right) \cap H P\left(v_{b}, v_{b+1}\right)=\varnothing$, so that $V\left(m_{a}, P\right) \cap V\left(m_{b}, P\right)=\varnothing$ where $m_{a}$ (resp. $\left.m_{b}\right)$ is the midpoint of $E\left(v_{a}, v_{a+1}\right)$ (resp. $E\left(v_{b}, v_{b+1}\right)$ ). By Lemma 3 we can conclude that the number of guard edges does not exceed two (see Fig. 11(b)). In a rectilinear polygon with rectilinear holes, there are no guard edges.

\section{Acknowledgments}

The first author would like to thank Dr. Jeong In Doh for helpful discussions and the anonymous referees for improving the readability of this paper.

\section{References}

1. A. Aggarwal, The art gallery theorems: its variations, applications and algorithmic aspects, $\mathrm{Ph} . \mathrm{D}$. Thesis, Johns Hopkins University, Baltimore, MD, 1984.

2. D. Avis and G. T. Toussaint, An efficient algorithm for decomposing a polygon into star-shaped polygons, Pattern Recognition 13 (1981), 395-398.

3. D. Avis and G. T. Toussaint, An optimal algorithm for determining the visibility of a polygon from an edge, IEEE Trans. Comput. 30 (1981), 910-914.

4. V. Chvátal, A combinatorial theorem in plane geometry, J. Combin. Theory Ser. B 18 (1975), 39-41.

5. H. Edelsbrunner, J. O'Rourke, and E. Welzl, Stationing guards in rectilinear art galleries, Comput. Vision Graphics Image Process. 27 (1984), 167-176.

6. S. Fisk, A short proof of Chvátal's watchman theorem, J. Combin. Theory Ser. B 24 (1978), 74.

7. R. Honsberger, Mathematical Gems II, Mathematical Association of America, Washington, DC. 1976.

8. D. T. Lee and A. K. Lin, Computational complexity of art gallery problems, IEEE Trans. Inform. Theory 32 (1986), 276-282.

9. D. T. Lee and F. Preparata, An optimal algorithm for finding the kernel of a polygon, J. Assoc. Comput. Mach. 26 (1979), 415-421.

10. J. O'Rourke, Galleries need fewer mobile guards: a variation on Chvátal's theorem, Geom. Dedicata 14 (1983), 273-283.

11. J. O'Rourke, Art Gallery Theorems and Algorithms, Oxford University Press, New York, 1987.

12. D. Penny, Perspective in Mathematics, Benjamin, Menlo Park, CA, 1972.

13. J. Sack, An $O(n \log n)$ algorithm for decomposing simple rectilinear polygons into convex quadrilaterals, Proc. 20th Allerton Conf, 1982, pp. 64-74.

14. J.-R. Sack and S. Suri, An optimal algorithm for detecting weak visibility of a polygon, IEEE Trans. Comput. 39 (1990), 1213-1219.

15. S. Y. Shin, Visibility and its related problems, Ph.D. Thesis, Department of Industrial and Operations Engineering, University of Michigan, Ann Arbor, MI, 1986.

16. S. Y. Shin and T. C. Woo, An optimal algorithm for finding all visible edges in a simple polygon, IEEE Trans. Robotics Automation 5 (1989), 202-207.

17. S. Suri and J. O'Rourke, Worst-case optimal algorithms for constructing visibility polygons with holes, Proc. 2nd ACM Symp. on Computational Geometry, 1986, pp. 14-23.

Received October 15, 1990, and in revised form February 26, 1993. 